\title{
Potential of Big Visual Data and Building Information Modeling for Construction Performance Analytics: An Exploratory Study
}

\author{
Kevin K. Han ${ }^{\mathrm{a}, 1, *}$, Mani Golparvar-Fard ${ }^{\mathrm{b}}$ \\ ${ }^{a}$ Assistant Professor, Department of Civil, Construction, and Environmental Engineering, North Carolina State University, \\ Raleigh, $\mathrm{NC}$ \\ ${ }^{b}$ Assistant Professor and Faculty Entrepreneurial Faculty Fellow, Department of Civil and Environmental Engineering, and \\ Department of Computer Science, University of Illinois at Urbana-Champaign, Urbana, IL
}

\begin{abstract}
The ever increasing volume of visual data due to recent advances in smart devices and camera-equipped platforms provides an unprecedented opportunity to visually capture actual status of construction sites at a fraction of cost compared to other alternatives methods. Most efforts on documenting as-built status, however, stay at collecting visual data and updating BIM. Hundreds of images and videos are captured but most of them soon become useless without properly being localized with plan document and time. To take full advantage of visual data for construction performance analytics, three aspects (reliability, relevance, and speed) of capturing, analyzing, and reporting visual data are critical. This paper 1) investigates current strategies for leveraging emerging big visual data and BIM in construction performance monitoring from these three aspects, 2) characterizes gaps in knowledge via case studies and structures a road map for research in visual sensing and analytics.
\end{abstract}

Keywords: Big Visual Data, Images, Videos, Point Cloud, Construction Progress Monitoring, Quality Control

\section{Introduction}

2 Various applications of camera networks on construction sites have received increasing attention in recent years. Some of the applications include progress tracking, safety monitoring, site security, and as-built documentation. Other recent developments are the diffusion of consumer commodity devices with built-in cameras, such as smart phones, tablets, wearables, and camera-equipped unmanned ground/aerial vehicles (denoted as UGV and UAV). These camera-equipped platforms have led to an exponential growth in the volume of images and videos that are being recorded on construction sites on a daily basis. To streamline the process of collecting images and videos, professional photography has also received significant attention. According to one of the popular construction documentation service providers, about 325,000 images are taken by professional photographers, 95,400 images by webcams, and 2,000 images by construction project team members at a typical commercial building project ( $750,000 \mathrm{sf})$ [1]. These are more than 400,000 images in total. The number can be much higher with the use of UGV and UAV for capturing images. The

\footnotetext{
${ }^{*}$ Corresponding author

Email addresses: kevin_han@ncsu.edu (Kevin K. Han ), mgolpar@illinois .edu (Mani Golparvar-Fard)

${ }^{1}$ Tel: +1 9195158719
} 
ever increasing volume of digital images provides an unprecedented opportunity to visually capture actual status of construction sites at a fraction of cost compared to other alternatives such as laser scanning.

In the meantime, $n^{\text {th }}$-dimensional (nD) Building Information Modeling (BIM) (i.e. 3D models enriched with performance information such as time, cost, safety, and productivity) has received a certain level of maturity. As BIM has advanced, enhanced 3D visualization with semantic building information at jobsites improved communication and coordination. The applications include design development, construction coordination, and planning and the value-added by BIM are well documented. For example, Lu et al. [2] report $6.92 \%$ cost saving by using BIM even after accounting for the added efforts during the design phase. Similarly, Staub-French and Khanzode [3] report 25-30\% productivity improvement through BIM-driven coordination and constructability reviews that identified most design conflicts prior to construction.

Most efforts on documenting as-built status stay at collecting visual data and updating BIM. Hundreds of images and videos are captured during construction but most of them soon become useless without properly being localized with plan document and time. There is a need for a mechanism that would localize a vast amount of visual data with respect to BIM so they can provide useful as-built status with accurate location. There is a need for a visual sensing mechanism that would automate information flow at a high frequency for capturing changes continuously, which requires more visual data to "infill" the gaps/disconnection among images and videos.

With the problems discussed above, what the AEC industry needs to solve using big visual data are: 1) data collection and analytics - visual data captured at a high rate of frequency and its analytics that provide insights on project controls; 2) reporting - effectively capturing and reporting issues found on construction sites; and 3) communication - efficient and effective flow of information within a construction site and site-to-office.

In principal, as-built documentation (images and videos) and $\mathrm{nD}$ BIM provide good opportunities for sensing, analysis, and communication of construction performance among project teams [4]. As-planned (e.g., nD BIM) and as-built (e.g., images, videos, and 3D point clouds) documents/models combined can support daily work execution and short-term planning (see Figure 1). The workflow shown in Figure 1 has an ongoing loop between the short-term planning and daily project controls. What have not been fully investigated by researchers are 1) dealing with images and videos that are not localized with respect to BIM automatically or with minimal input from a user (see Figure 2) and 2) dealing with a large amount of visual data that accumulates over time. This paper aims to tackle these challenges and provide a roadmap for using big visual data that is well-suited for construction performance monitoring applications.

The main challenges associated with localization of images with respect to BIM include 1) directly aligning images to BIM, 2) aligning via reconstructing a 3D point cloud from image collections, and 3) enhancing alignment using BIM as a constraint to improve 3D reconstruction. This paper also investigates the potential of using the resulting integrated information model to visually identify location and orientation of a user in 3D and provide information query and content authoring capabilities (see Figure 1). Finally, techniques for comparison of the images and/or the produced point clouds vs. BIM are investigated. Using a large pool of case studies and datasets collected, the underlying technical problems and perceived opportunities in the three areas above are thoroughly explored. In each case, the capturing, analyzing, and reporting the visual performance data are investigated from the following three perspectives:

1. Reliability-accuracy and completeness of the collected data. 2. Relevance- usefulness to short-term planning (e.g. three-week lookahead plans). 3. Speed-collecting, analyzing, and reporting at a pace that enables decision making in coordination meetings.

In summary, the contributions of this exploratory study are: 1) investigating current strategies for leveraging emerging big visual data and BIM in construction performance monitoring; and 2) characterizing 


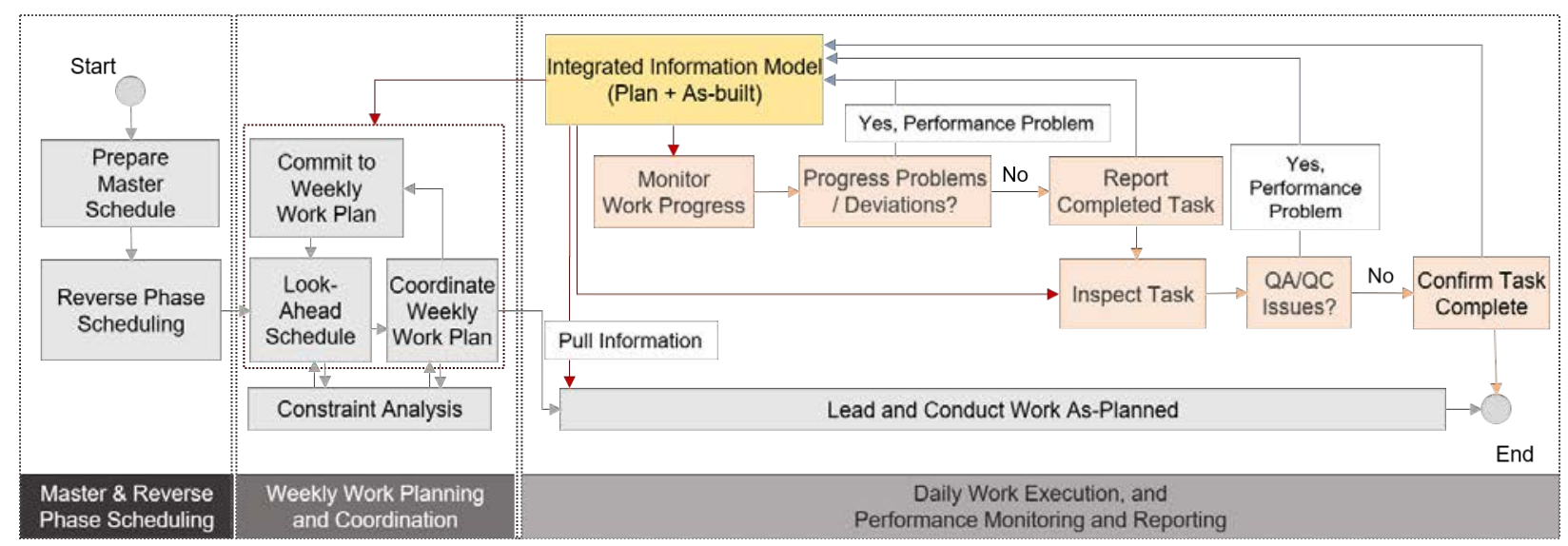

Figure 1: The Integrated Project Model for supporting short-term planning and daily execution and reporting. Adapted from [4].

gaps in knowledge via case studies and structuring a road map for research in visual sensing and analytics.

\section{Defining Big Visual Data for Construction Performance Monitoring}

According to National Consortium for Data Science in 2012, "big data" or massive data collections is historically transforming every sector of the society - business, science, medicine, and government [5]. Big data is a broad term with multiple definitions but there are common characteristics. Most commonly used characteristics that define big data are volume, variety, and velocity or $3 \mathrm{~V}$ model [6]. There are other emerging "Vs" that are defined by different data scientists, such as veracity and variability [6-8]. Veracity is not directly applicable to visual data because visual data capture as-is conditions. They capture scenes and objects how they appear to be. They can certain have bad qualities (i.e., blurriness) and misinterpretations but they still present $a s$-is conditions. Text and words can have varying meanings and change their meanings as time evolves [8]. These characteristics of variability, however, are not applicable to visual data because the contents (what is captured in pixels) do not change. Again interpretation of an object captured might change but not the values of pixels representing the object. The pixels representing an object form features that are used for visual data analytics. In addition, big visual data have to be characterized differently because the use of database is not directly applicable for detecting meaningful patterns out of visual data. The only way to place visual data in form of tables is to put attributes of exchangeable image file (EXIF) tags or place analyzed data (already processed). There is no existing database solution that can capture and provide meaningful real-time querying of raw visual data at the best of the authors' knowledge.

The $3 \mathrm{~V}$ model, however, is still applicable for characterizing big visual data. Volume can characterize visual data for each construction project because of the large volume of visual data accumulated over the duration of construction. Visual data collected during daily execution (see Figure 1) can be in a scale of terabytes (refer to case studies in the following section). For a company to manage hundreds of their projects, the volume of their visual data can grow to petabytes. Variety also characterizes big visual data because they come in various formats. For instance, there are images and videos with different formats. Different types of camera lens require different processing (i.e., fish lens requiring careful calibration to remove radial distortion). Lastly, velocity can characterize visual data because of continuously accumulated images throughout construction. All project participants - owners, general contractors, and subcontractors capture visual data every day for multiple purposes. 


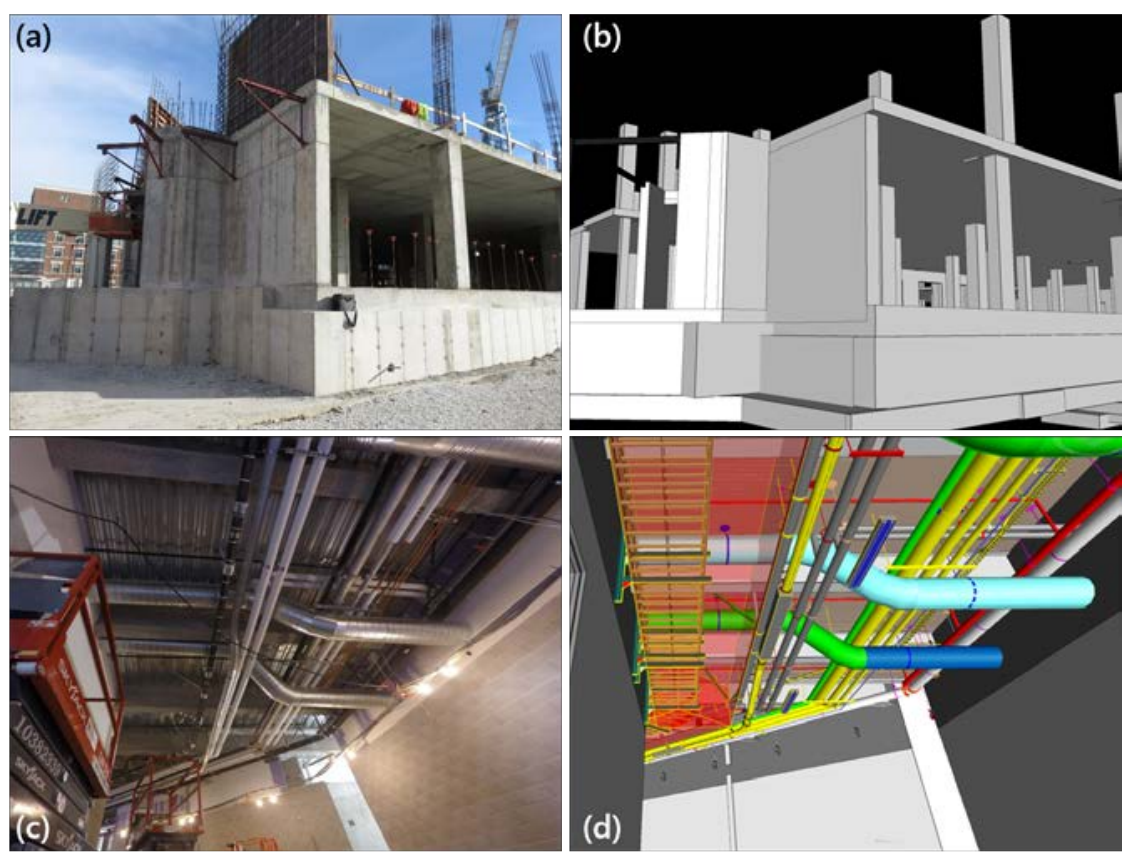

Figure 2: Side-by-side comparison of a site image and BIM for both exterior and interior spaces. The goal is to identify location and orientation of the image with respect to the model automatically or with minimal user input to allow for scalability.

In addition to the $3 \mathrm{~V}$ model, what can characterize big visual data for construction performance monitoring is incremental time instances. To monitor construction performance, a gradual change throughout construction must be captured. Performing vision-based visual analytics require filtering or gathering of a set of images that are captured at a given time. This paper refers to it as "a time instance." Each set of images captured at a time instance will be analyzed for predicting performance. Each project will have many time instances throughout its construction phase. For example, a time instance can have tens to thousands of images that are analyzed. A project can have tens to hundreds of thousand images from all these time instances.

Therefore, big visual data in this paper refers to hundreds of thousand images that are collected throughout a construction project. The analytics refers to each analysis conducted on each time instance. The hypothesis in this paper is that the predicted performance from these time instances can be compared against 4D BIM over time for detecting performance deviation and improving communication through visualized performance deviation. The next two sections discuss challenges associated with collecting and maintaining big visual data (Section 3) and cover challenges and practical benefits associated with conducting individual analyses of time instances over a period of construction (Section 4).

\section{Gaps in Knowledge in Collecting and Maintaining Big Visual Data}

Big visual data require a systematic way of collecting and maintaining data. A file size of an image varies from a few hundred kilobytes (KB) to a few megabytes (MB) depending on its resolution. Hundreds of thousand images can add up to gigabytes (GB) to terabytes (TB). To help visualize what it means to have big visual data, the authors have summarized collected data for three short-term pilot projects. These were conducted during actual construction of three different projects and therefore had limited and different durations. They consist of a stadium, university residential hall, and hotel projects. 
A UAV and a UGV were used in the stadium project (SP) for 11 months. A UAV and point-and-shoot cameras were used in the residential hall project (RP) for 6 months. Only UAV was used in the hotel project (HP) for 2 months. The collected data does not include images that are captured as part of daily practices. There is no existing tool that aggregates these images from all project participants, which may require significant coordination in the beginning of a project. This is also one of the challenges associated with sharing all visual data captured during construction.

Table 1 and Table 2 summarize the collected visual data. The data sizes for SP, RP, and HP are 105 GB, $141 \mathrm{~GB}$, and $24 \mathrm{~GB}$, respectively. What really matters is the data size per time instance shown in Table 2: about 4.0 GB, 5.2 GB, and 2.4 GB for SP, RP, and HP, respectively. The total number of time instances dictates the data size. For instance, if data are collected at a higher frequency (e.g., a few times a week), the total data size can be much higher at the end of construction. The numbers presented in these two tables only reflect raw visual data. What make them "big" are the visual analytics conducted on them, which require a much larger storage space and heavy computing. For instance, a typical 3D reconstruction may require two to four times larger storage space and also create two to three times more files depending on 3D point clouds resolutions and how data are saved.

Table 3 summarizes output of visual data analytics conducted on HP. HP was carefully coordinated from the beginning for consistent and frequent visual data analytics. There were some variables that affected the quality of data capture due to site and weather conditions (i.e., crane locations and rain) but the data capture and the performed analysis (only 3D reconstruction in this case) were fairly consistent every week for two months. As seen in the figure, the data size is about $430 \mathrm{~GB}$ with the average of $43 \mathrm{~GB}$ per time instance. Given that this project's estimated duration is 144 weeks, the projected file size is about $6.2 \mathrm{~TB}$. The only kind of processing done for this dataset was 3D reconstruction. With more visual analytics such as [9] and [10], the file size can substantially increase. To take full advantage of these data for project controls, change in time (similar to 4D for BIM) should be captured and visualized as 4D point clouds (multiple point clouds at multiple time instances). 4D point clouds aligned with 4D BIMs can support short-term planning sessions by a web-based tool presented in [11]. These numbers can increase significantly based on any further visual analytics, such as object recognitions for progress monitoring [9] and activity analysis [10].

The best practices for managing and sharing files during construction include file managers that are cloud-based or the file transfer protocol (FTP) using servers. Either ways, these currently available solutions are not designed for dealing with thousands of files within a folder (e.g., one time instance per folder) and are not suitable for quick inspection, visualization, and filtering of a long list of visual data. Moreover, in the research community, big visual data have been focused on processing a large set of datasets in a lump-sum manner (e.g., ImageNet [12] and PASCAL Visual Object Classes (VOC) [13]). The research community in the AEC industry does not have a comprehensive visual dataset that is designed for the applications discussed in this paper. The first attempt was Construction Material Library (CML) created by Dimitrov and Golparvar-Fard [14] but more groups of researchers and practitioners have to work together to continuously improved CML before it can be used as a benchmark.

\section{Gaps in Knowledge in BIM-driven Visual Data Analytics}

Today, almost all personnel on a jobsite are familiar with cameras and they are readily available to capture images and videos. The principal problems lie in site conditions that cause difficulties in finding suitable positions for time-lapse cameras that minimize occlusions [15], proper locations and orientations for taking images via smart devices [16], or identifying areas that need to be scanned via camera-equipped UAVs [17]. A general rule when it comes to placement of time-lapse cameras is that the instrument should be considerably higher than the work being recorded. They should also capture a wide field of view in order to 


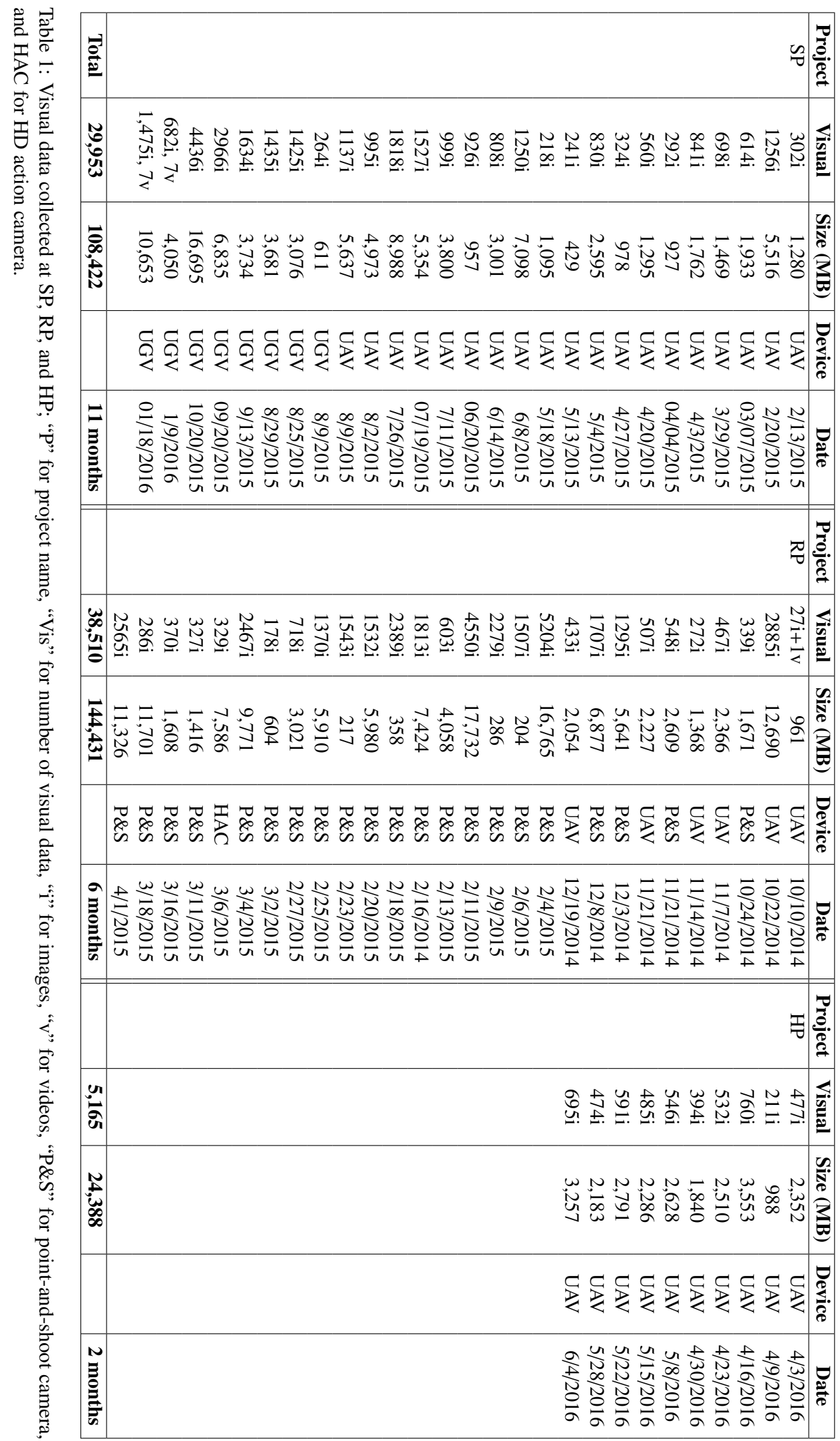




\begin{tabular}{|c|c|c|c|}
\hline Project & \# of instances & images per instance & File size per instance \\
\hline SP & 27 & 1,109 & $4,016 \mathrm{MB}$ \\
RP & 28 & 1,426 & $5,158 \mathrm{MB}$ \\
HP & 10 & 517 & $2,439 \mathrm{MB}$ \\
\hline
\end{tabular}

Table 2: Data collection summarized by time instances.

\begin{tabular}{|c|c|c|c|}
\hline Date & Images & Files & Size (GB) \\
\hline $4 / 3 / 2016$ & 477 & 73,899 & 14.4 \\
$4 / 9 / 2016$ & 211 & 2,416 & 5.0 \\
$4 / 16 / 2016$ & 760 & 17,150 & 73.9 \\
$4 / 23 / 2016$ & 532 & 12,045 & 124.0 \\
$4 / 30 / 2016$ & 394 & 4,461 & 26.6 \\
$5 / 8 / 2016$ & 546 & 6,140 & 41.9 \\
$5 / 15 / 2016$ & 485 & 5,990 & 37.0 \\
$5 / 22 / 2016$ & 591 & 6,545 & 40.4 \\
$5 / 28 / 2016$ & 474 & 5,243 & 26.8 \\
$6 / 4 / 2016$ & 695 & 7,820 & 41.1 \\
\hline Total & $\mathbf{5 , 1 6 5}$ & $\mathbf{1 4 1 , 7 0 9}$ & $\mathbf{4 3 1 . 2}$ \\
\hline
\end{tabular}

Table 3: HP after 3D reconstruction; "Images" for the numbers of images and "Files" for the numbers of files.

minimize occlusions by foreground activities or other obstructions. These considerations can result in a better perspective, clarity in physical relationships, and larger image surfaces for construction monitoring purposes. Some of these conditions apply to ground cameras as well. In the case of using smartphone images, a complete view of an area or an object of interest has to be captured by multiple images. For stitching these images together or producing 3D point clouds (whether captured via smart devices or UAVs), all images should have a certain amount of overlaps, which is an additional constraint for which needs to be accounted.

To analyze construction performance, an as-is condition needs to be compared to an as-planned condition. One way to achieve this is to align visual data with BIM, which involves manual work - e.g., selection of correspondences between visual data and BIM or placement of surveyed targets (ground control points) for "automated registration." The alignment process is a very important challenge for big visual data because big visual data analytics would not be feasible without a way to scale up this alignment process by automation or even semi-automation. Therefore, this section highlights challenges associated with 1) alignment of visual data to BIM under various conditions and 2) various aspects of visual data analytics.

\subsection{Image-to-BIM alignment}

\subsubsection{Time-lapse images and videos from fixed locations}

Using fixed cameras - with or without Pan/Tilt/Zoom capability - for providing live-stream video feed or time-lapse images of a jobsite is very common nowadays. Most of the literature on time-lapse photography for construction monitoring mostly focus on visualization of ongoing operations, improving situational awareness (e.g. for crane operations), documentation (e.g. progress changes, claims analysis, and accident investigation) [18-20] and visualizing progress deviations against BIM [15, 21-23]. One of the earlier examples is Abeid et al. [18, 19] which developed Photo-Net and PhotoNet II that visualize time-lapse images at varying times tied with schedules and weekly cumulative percentages of progress. In most of these 
solutions, users can see the current schedule with the accumulated progress and the physical appearance at a certain time captured by fixed cameras, with or without a reference to the planned progress. Similarly but with enhanced visualization through use of 4D BIM, [15, 21-23] present time-lapse photographs aligned with BIM. They present progress by intuitively visualizing deviations via 4D BIM instead of showing only Gantt charts. Time-lapse images are calibrated with known 3D locations of BIM for the alignment. Instead of graphs and percentages, progress is reported using color-coded BIM elements, providing an effective mechanism for associating element-level progress deviations in 3D.

There are four key challenges associated with using time-lapse images and BIM for construction performance monitoring:

(1) Need for automated registration and alignment of time-lapse images-Cameras used for time-lapse images are subject to lateral movements. Their viewing directions and fields of view change from time to time. These images need an algorithm that can automatically align time-lapse images with their subtle changes. This can be done via automated feature detection, matching, and homography transformation [24] between two sequential images. For instance, Image B followed by Image A needs to be warped into Image B's viewpoint. Nevertheless, varying lighting and shadow conditions can have a significant impact on detected features and their descriptors can create a different set of features. This problem still exists, despite the promise of most rigorous feature detection techniques such as Scale-invariant Feature Transform [25] which is designed to be invariant to such changes. For instance, a strong shadow is casted by a steel structure on the right in the upper image of Figure3. Moreover, color intensity varies widely from the upper image to the lower image in the same figure. It is also possible that a fixed camera moves overtime due to multiple factors (e.g., wind, earthquake, and vibration) as shown in Figure 4. As can be seen from the red circles in this figure, the camera is slightly rotated over time. The visual analytics techniques discussed previously $([18,19,21,26]$ only localize the initial image frame and changes in appearance (e.g., structure and lighting and shadow) may challenge its registration progress. Registering subsequent image frames can deal with this challenge and requires feature detection and matching, ensuring that localization needs to happen only once in the beginning.

(2) $3 D$ to $2 D$ registration - Backprojecting BIM elements onto a time-lapse image requires camera calibration information. This means both camera intrinsic parameters (focal length, skew parameter, and the coordinates of the optic center) and extrinsic parameters (rotation and translation) are required. Camera calibration [24] provides an opportunity to identify camera parameters though this requires a camera calibration rig (a gridded board similar to a chessboard) to be formed and placed on a jobsite. A more common approach is for a user to select 2D features in an image, identify the correspondences with 3D points in the scene, and then solve for Perspective-n-Point algorithm [27]. Given a possible change in a viewpoint and a field of view, this process has to be frequently repeated to guarantee successful registration if sufficient overlaps among images are not present. However, with this user input, registration is always guaranteed and a small set of images can be easily registered for various useful applications - e.g., time-lapse rendering, annotation, and documentation.

(3) Limited visibility per element- Time-lapse images and videos from fixed locations always have challenges associated with occlusions and limited visibility $[15,18]$. As can be seen in Figure 5, a single viewpoint causes all elements to have limited visibilities, simply because they are seen from one location and orientation.

(4) Need for multiple viewpoints for maximizing coverage-Other than installing more fixed cameras for more views, Degol et al. [28] propose autonomous installment of wireless cameras using UAVs. A UAV carries a wireless camera that can be mounted underside of a steel beam. It moves the wireless camera and mounts it to different locations. This approach ensures multiple fixed viewpoints for the maximized 


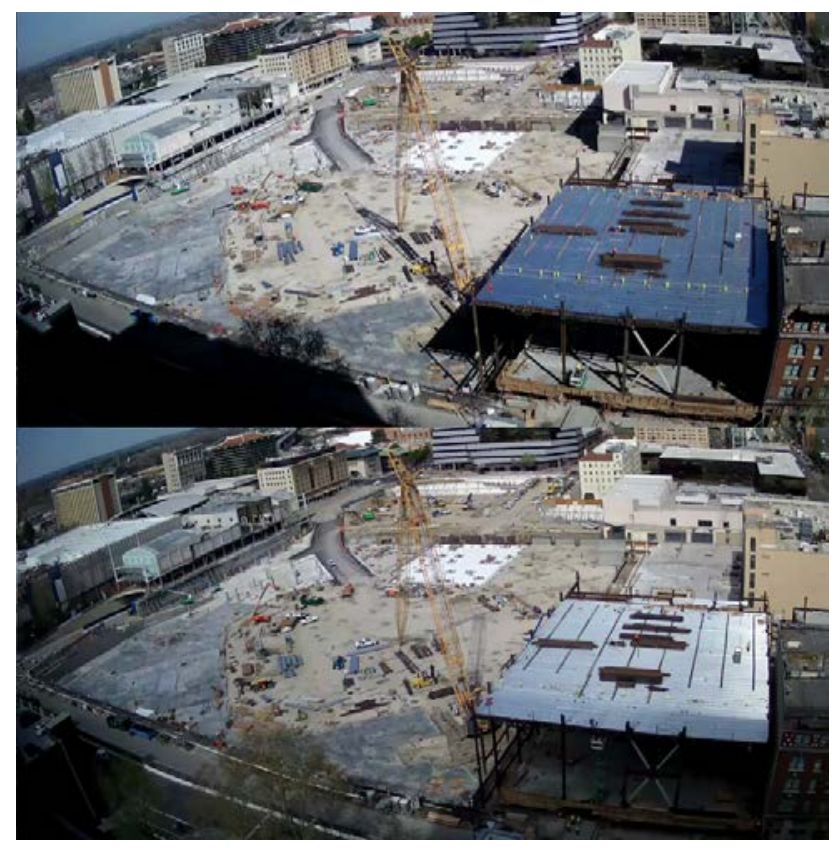

Figure 3: Two still frames of a time-lapse video captured at 11:36am (top) and 1:38pm (bottom) on the same day.

coverage. Their experiment is performed in a controlled environment. Implementing this system in a uncontrolled environment with many obstacles (i.e., construction sites) is going to be a challenge. Moreover, manual installment of fixed cameras have multiple challenges - e.g., ensuring clear viewpoints, operation and maintenance, and limited areas for installment.

\subsubsection{Unordered set of images}

Images and videos collected by digital cameras and smartphones are taken at various locations by field personnel, including superintendents, project engineers, and subcontractors. To streamline the process, owners and general contractors also hire construction documentation service providers or use cameraequipped UAVs to professionally take images and document them. In the former case, images are typically uncalibrated and their locations and orientations are unknown. This makes it very hard to accurately localize them with BIM. When images are collected via professional photography, locations and orientations of where images are captured from are manually documented. In the case of images taken with camera-equipped UAVs, GPS coordinates and camera orientation are known in most cases. Even in these conditions, it is still very difficult and is tedious to find the exact views in BIM for 2D to 3D comparison, especially for Mechanical/Electrical/Plumbing (MEP) systems. This is due to inaccurate GPS coordinates especially in the vertical axis. In the case of using ground control points (GCP), an accurate alignment can be achieved. However, it requires installment of the ground targets that are pre-surveyed by experts. Moreover, BIM is not geo-referenced in many cases. This means that BIM has its own coordinate system and manual registration is inevitable.

Aligning an unordered set of images with a BIM can be done by creating a point cloud (SfM) and selecting correspondences between the point cloud and BIM. Similarity transformation -with seven degrees of freedom- between two selections brings two models into an alignment by solving a closed-form solution of absolute quaternion [29-31] (see Figure 10). Alternatively, model-assist SfM [27] can align an initial camera with a BIM in the beginning and create a point cloud that is aligned with the BIM by solving a PnP 


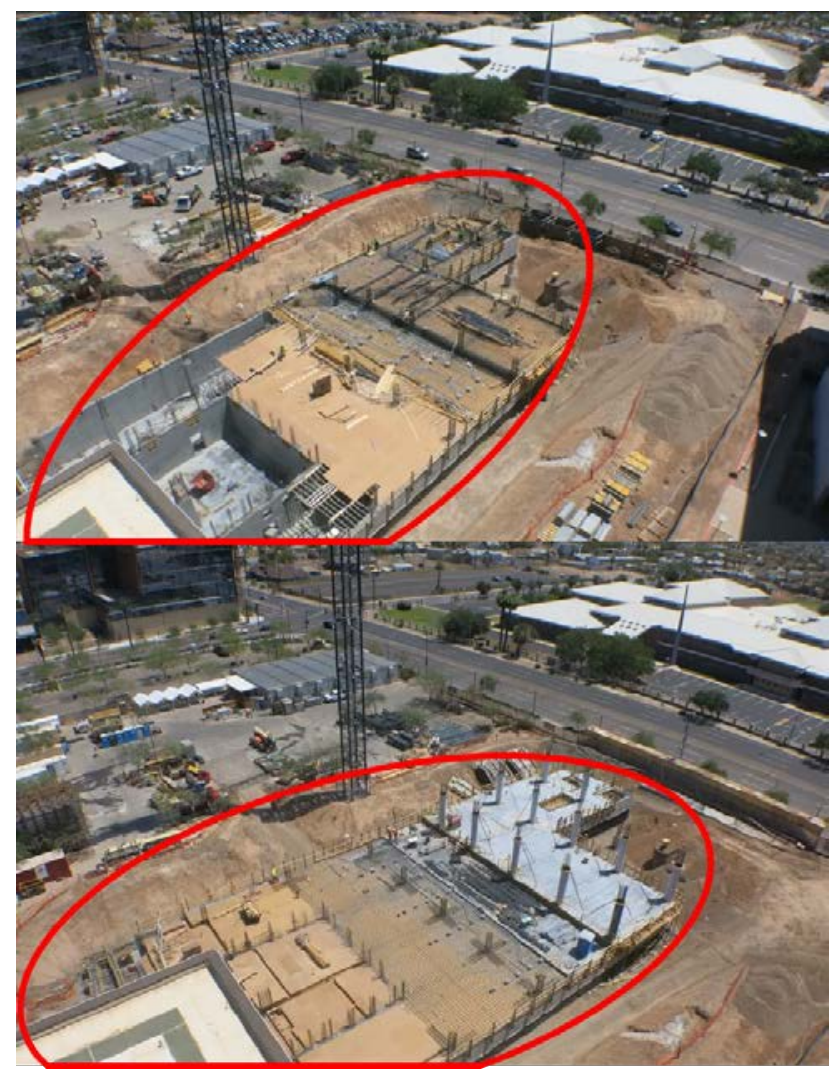

Figure 4: Two still frames of a time-lapse video showing change in camera rotation.

problem (see Figure 11. Once the as-built point cloud and BIM are aligned, all images registered during the $3 \mathrm{D}$ reconstruction procedure are aligned with the BIM. Main challenges of the former (create a point cloud first and then align with the BIM) are 1) there is no guarantee on completeness of the point cloud, 2) having sufficient overlaps among images covering areas of interest are required, and 3) interacting with the BIM and point cloud in a 3D environment when selecting correspondences is often difficult.

Model-assisted SfM, on the other hand, guarantees completeness of the point cloud through creating anchor cameras (cameras that are manually registered by users - e.g., the initial camera) whenever there are insufficient overlaps and making selections on 2D images is much easier than doing it on 3D point clouds. However, a user still needs to manually interact with the BIM in a 3D environment for selecting correspondence, which is difficult for a complex structure. Open research challenges, therefore, include automated selection of an initial anchor camera and automatic registration of the subsequent anchor cameras to the BIM.

\subsubsection{Sequenced set of images}

A sequenced set of images typically guarantee sufficient overlaps required for image-based 3D reconstruction. It, however, still has challenges - e.g., no guarantee on completeness of the reconstruction, drift (accumulated error when a structure has a wide baseline), plane surface deflection, and unit-less as-built models before being registered with BIMs (see Figure 6). For the case of images taken by UAVs, synced GPS coordinates can be used for aligning BIMs with point clouds. However, as mentioned previously, inaccurate GPS coordinates especially in the vertical axis and BIMs not in the world coordinate system are 


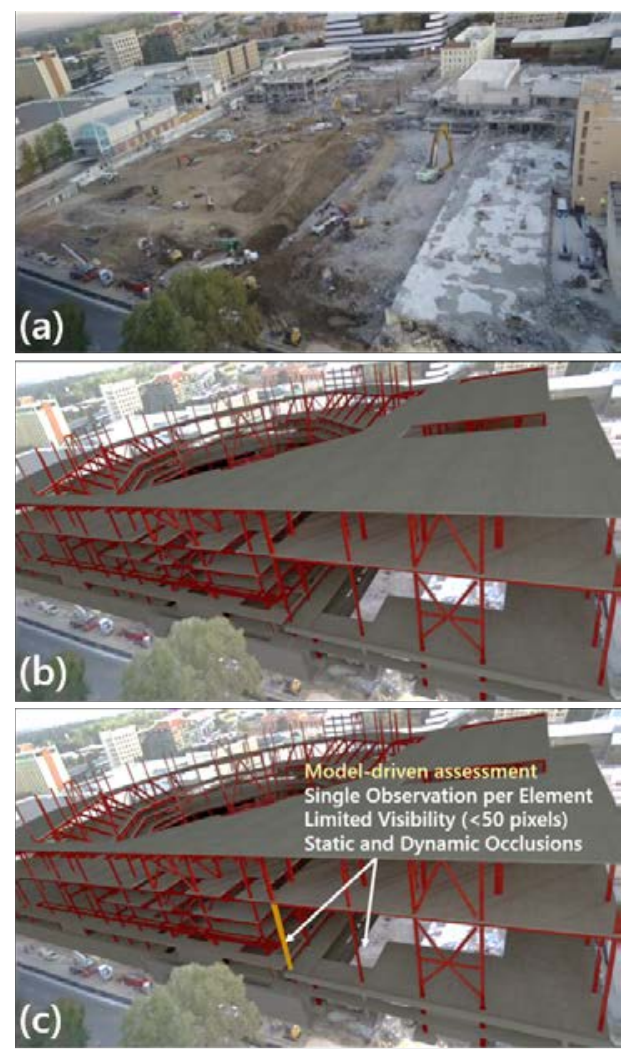

Figure 5: (a) a picture taken with a time-lapse camera; (b) 4D BIM is overlaid on the picture; (c) highlighting some of the challenges of using a single view for model-driven production monitoring.

still unresolved challenges. Moreover, abovementioned challenges associated with as-built models being unit-less also applies to these images. Leveraging BIMs as a priori geometry can potentially solve these issues but is still an open research challenge.

\subsubsection{Radial distortion}

Radial distortion is a common challenge that all three types of images discussed above are facing. For aligning 2D images to BIMs, images should be undistorted to show well-aligned images with BIM. Radial distortion can cause incomplete and inaccurate point cloud generation. In these cases, 3D point cloudto-BIM alignment is not possible. Therefore, calibration is critical for 3D reconstruction of the distorted images. The standard way of calibrating a camera involves a manual process that uses a rig. It only needs to be done once if a camera zoom is fixed. Automated calibration still is an open challenge, especially for a single view image (i.e., time-lapse and individual images).

\subsection{Point cloud-to-BIM alignment}

As mentioned before, image-based 3D reconstruction does not guarantee completeness. Even if modelassisted SfM is used, image-based 3D point clouds are generally noisier than laser scanned point clouds and they are more affected by unwanted objects (e.g., backgrounds and objects that are not the structure of interest) constructed as part of a point cloud.Therefore, automated registration is harder compared to the methods proposed for laser scanned point clouds [32-34]. 

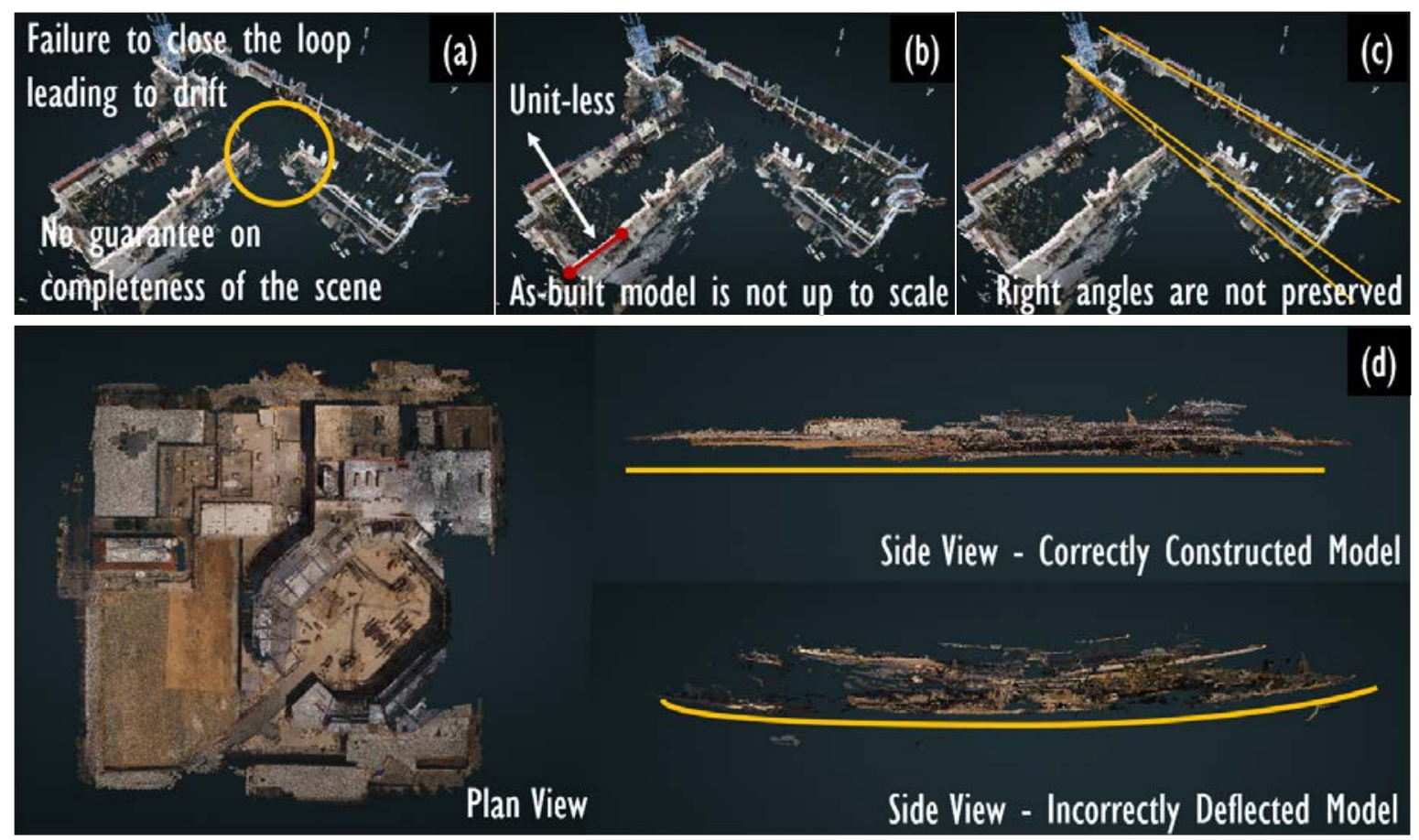

Figure 6: Challenges: a) drift and incompleteness; b) unit-less as-built model; c) right angles not being preserved; d) deflected surface

If pre-surveyed control points are used, correspondence selection is not required both for laser scanned and image-based point clouds because similarity transformations can bring point clouds to alignments with BIMs using the surveyed coordinates. It is worth noting that there are many instances of the surveying targets not being detected automatically due to not having clear views of the targets with enough resolutions. In this case, manual selection of correspondences from point clouds is required for performing similarity transformation. In case of using camera-equipped UAVs, the GPS coordinates synced with images can bring point clouds to alignments with BIMs. This process requires BIMs to be in the world coordinate system (typically Universal Transverse Mercator (UTM)). However, according to the authors' experience with the point clouds generated by well established commercial software and companies, the quality of this method is not consistent, although claimed to have a "centimeter" accuracy.

\subsection{Ubiquitous interaction with as-built and BIM}

To fully take advantage of model-driven analytics, users need to have access to as-built models and BIMs on- and off-site. Lin et al. [35] propose a web-based interface that visualizes as-built point clouds and BIMs together. It can also visualize image-to-BIM alignment for all images that are used for 3D reconstruction. Bae et al. [16] propose a mobile virtual reality system that can visualize and make 3D annotations on images that are part of a point cloud. A user cannot see the actual point cloud but can see the registered images and create annotations. This system allows users to access project information any places where users have access to Internet via mobile devices, such as tablets and smartphones. A major open challenge is interacting with the visualized model on these small mobile devices. Navigating in a 3D environment without a keyboard, mouse, and big screen is very difficult. Moreover, as-built models consist of large files 
(e.g., hundreds of images, point clouds, and BIMs that are hundreds of megabytes to gigabytes in size). Not all mobile devices have enough computing power to render and store them.

\subsection{Object detection}

So now an as-built model is aligned with a BIM but what does it tell us other than providing a nice visual? Object recognition methods enable useful analytics that provide insights for project management. For instance, there are many progress detection methods that detect BIM elements presented in as-built models. There are studies focusing on detecting geometry of BIM elements using laser scanning technique[36-39] and using image-based 3D reconstruction [30]. Only looking at geometry does not provide any insights on semantics of BIM elements. They cannot differentiate operation-level activities as long as points are present. For instance, these methods cannot differentiate a formwork, waterproofing panels, and finishing materials from concrete as long as a point cloud maintains the same geometry.

To overcome this limitation, there have been studies leveraging appearances of materials in as-built models and comparing them against material information stored in BIMs [9, 39]. In these studies, images are segmented by BIM elements for inferring progress. [39] use image masks to filter each element portion in an image for the progress inference. [9] extract an image patch per element and use its appearance (e.g., texture and HSV as features) for creating multiple discriminative material classification model for inferring progress. Studying interconnectivity relations of BIM is particularly useful for abovementioned visualbased progress detection [40, 41]. It can help make inferences on progress when an element of interest is not visible from the collected images.

Quality assurance and control (QA/QC) also can benefit from object detection. Laser scanning techniques are popular methods for this application due to highly reliable accuracies. Point clouds generated from these techniques and image-based 3D reconstruction also mainly examine geometry (i.e., plumbness and flatness) for quality inspection [38, 42-44]. These techniques both for progress detection and QA/QC largely depend on point cloud and image quality - limited views, completeness, and data collection frequency (see Figure 7). Expensive cost and time are also common challenges. Image-based 3D reconstruction and machine learning algorithms can take hours to run even with a high-end computing power and laser scanning operation can take hours of experts' labor.

There are many studies that leverage visual data for detecting safety hazards. Detecting safety hazards requires detection of objects that are related to safety. For instance, studies include detecting workers not wearing hard hats by face and hard hat color detection [45], unsafe action recognition of workers [46, 47], detecting workers and equipments [48-50], and detecting spatial safety hazard areas using laser scanning techniques [51, 52] and images [53]. These vision-based techniques also share the same challenges discussed for progress detection and QA/QC. In addition, they largely depend on where and how visual data is collected (i.e., viewpoints) and how fast they can detect and report safety hazards. After all, detection and reporting needs to happen before any fatality happens. Another challenge is that often safety related temporary structures are not modeled in BIM. This means that there is no expected state that can be inferred from BIM, which makes it harder to detect such structures (see Figure 8).

\subsection{Agent localization}

Agent localization is critical to data collection, analytics, and reporting. By agent, the authors meant data collectors such as field personnel, UGVs, UAVs and etc. Localizing UGVs and UAVs are critical for autonomous navigation. Simultaneous localization and mapping (SLAM) is a core area of research for autonomous navigation. SLAM, however, is subject to having drift, especially when areas of interest have large baselines. BIM, as a priori geometric information, can be potentially used for exterior and indoor 


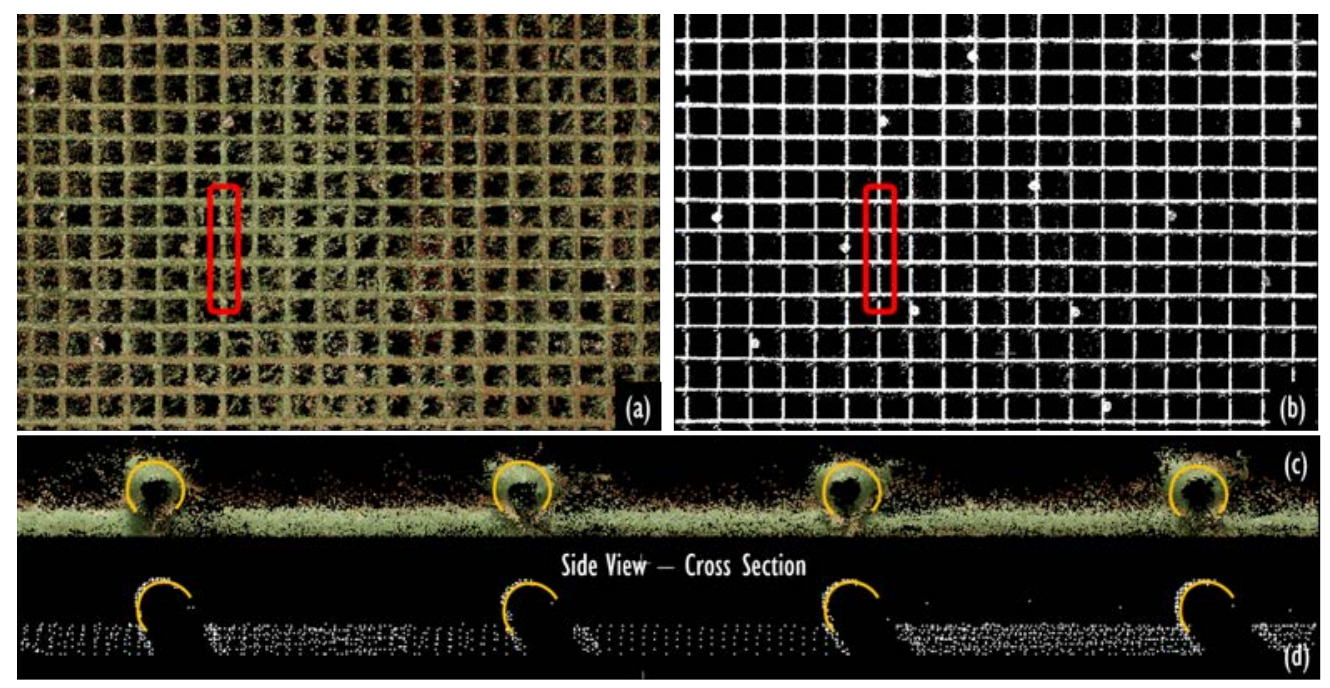

Figure 7: Challenges of limited views for QA/QC using point clouds: image-based 3D reconstruction - a \& c; laser scanned point cloud - b \& d.

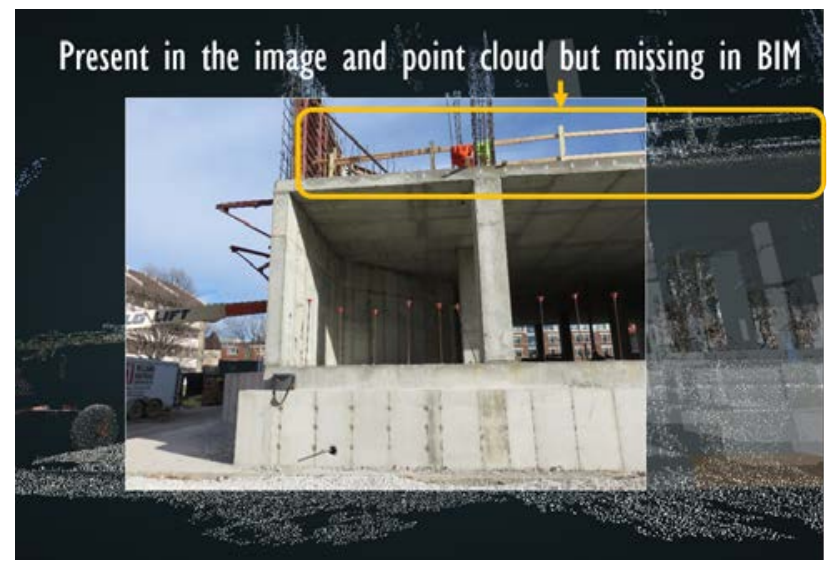

Figure 8: Challenge associated with detecting safety hazard - presented in images and point cloud but missing in BIM.

SLAM, correcting or calibrating positions of agents with BIM [17]. However, such method still remains as an open research question.

Agent localization can be used for virtual reality experience, especially for mounted displays (e.g., HoloLens and Oculus). Instances of display (snapshots) of what a field engineer sees can be used for quick annotation and reporting for all kinds of applications in construction, including communication of issues on progress, quality, and safety.

Visual data localized with BIM can support both field-to-office and office-to-field communication. Once all images are localized with a BIM, camera poses are recovered for all images with respect to the BIM's coordinate system, which allow localization with all BIMs (in the same coordinate system) used for the same project. Therefore, these images can be used for reporting and documentation, allowing effective twoway communication with visual aid between field and office. The following section presents visual data localization methods and its applications using real world case studies. 


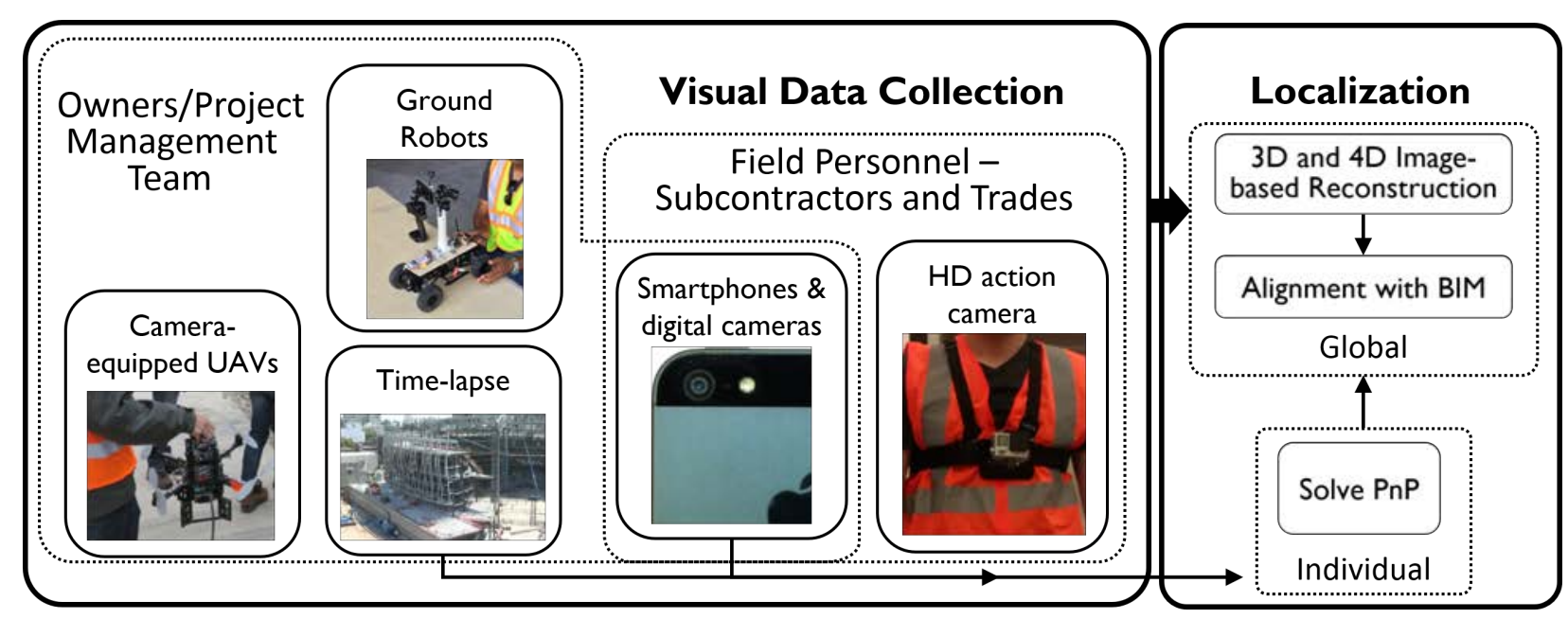

Figure 9: Overview of visual data collection and localization of the collected data with BIM.

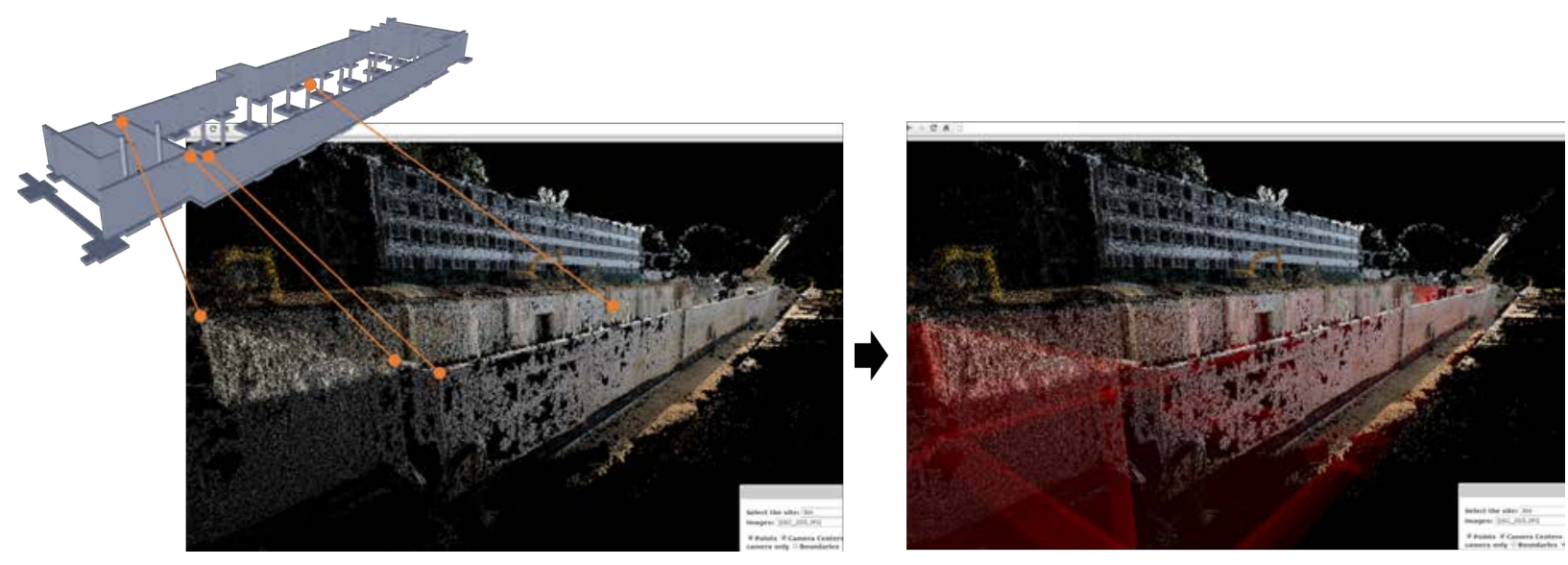

Figure 10: Global localization of images with a BIM through registering a point cloud with the BIM. Selection of corresponding feature (left) and registration with the BIM (right).

\section{Highlighting Opportunities with Big Visual Data in Construction}

Image-based 3D localization with BIM leads to unprecedented opportunities for new communication, taking advantages of both 2D and 3D visualization. This section presents these new opportunities with real world case studies - hospital, university, and sports facility projects. Images used in this experiment were taken by a UAV, UGV, and field personnel. For each application discussed in this section, both global and individual localizations with BIM are applicable and thus presented.

The high-level overview of data collection and localization is presented in Figure 9. Visual data collection can be done by 1) owners and project management teams (denoted throughout as OPM) and 2) field personnel such as subcontractors and trades.Visual data collected by OPM tend to focus on high-level activities and overall progress and structure. Possible methods of data collection by OPM include cameraequipped UAVs, UGVs, time-lapse or live videos at fixed locations, digital cameras, and smartphones. On the other hand, data collected by field personnel tend to focus on low-level activities and issues of their own scopes. Possible data collection methods include digital cameras, smartphones, and mounted action 

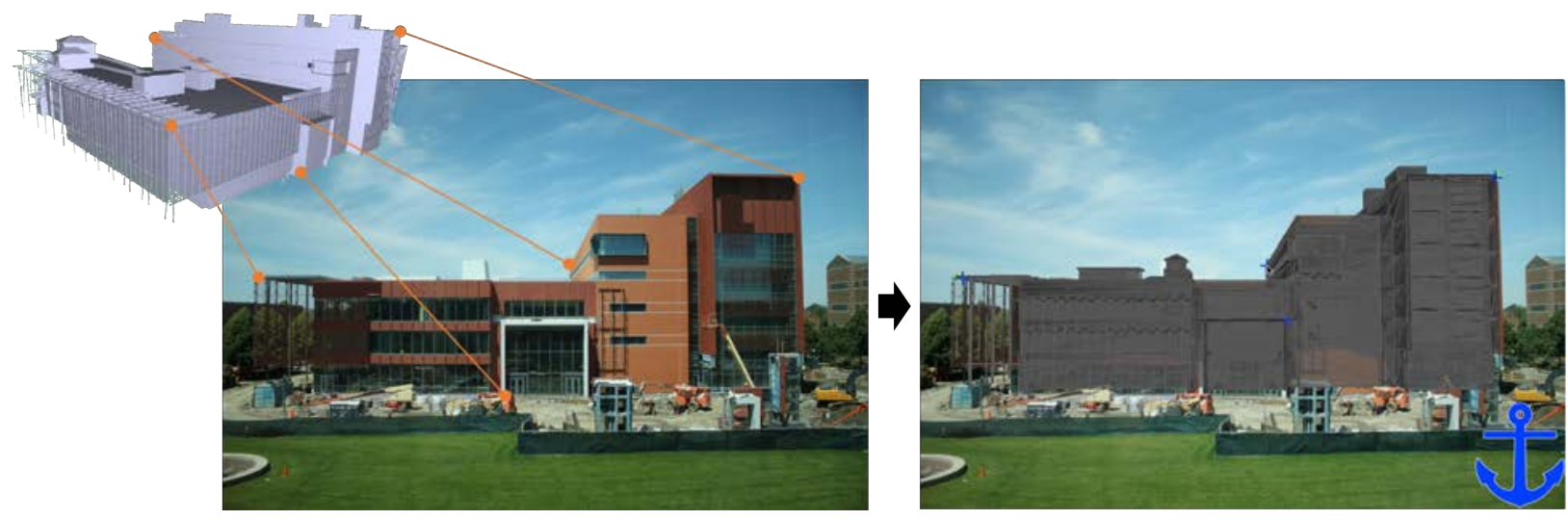

Figure 11: Individual localization of an image with a BIM - also a step in model-assisted SfM. Selection of corresponding feature (left) and calibration with the BIM (right). Adapted from [54]

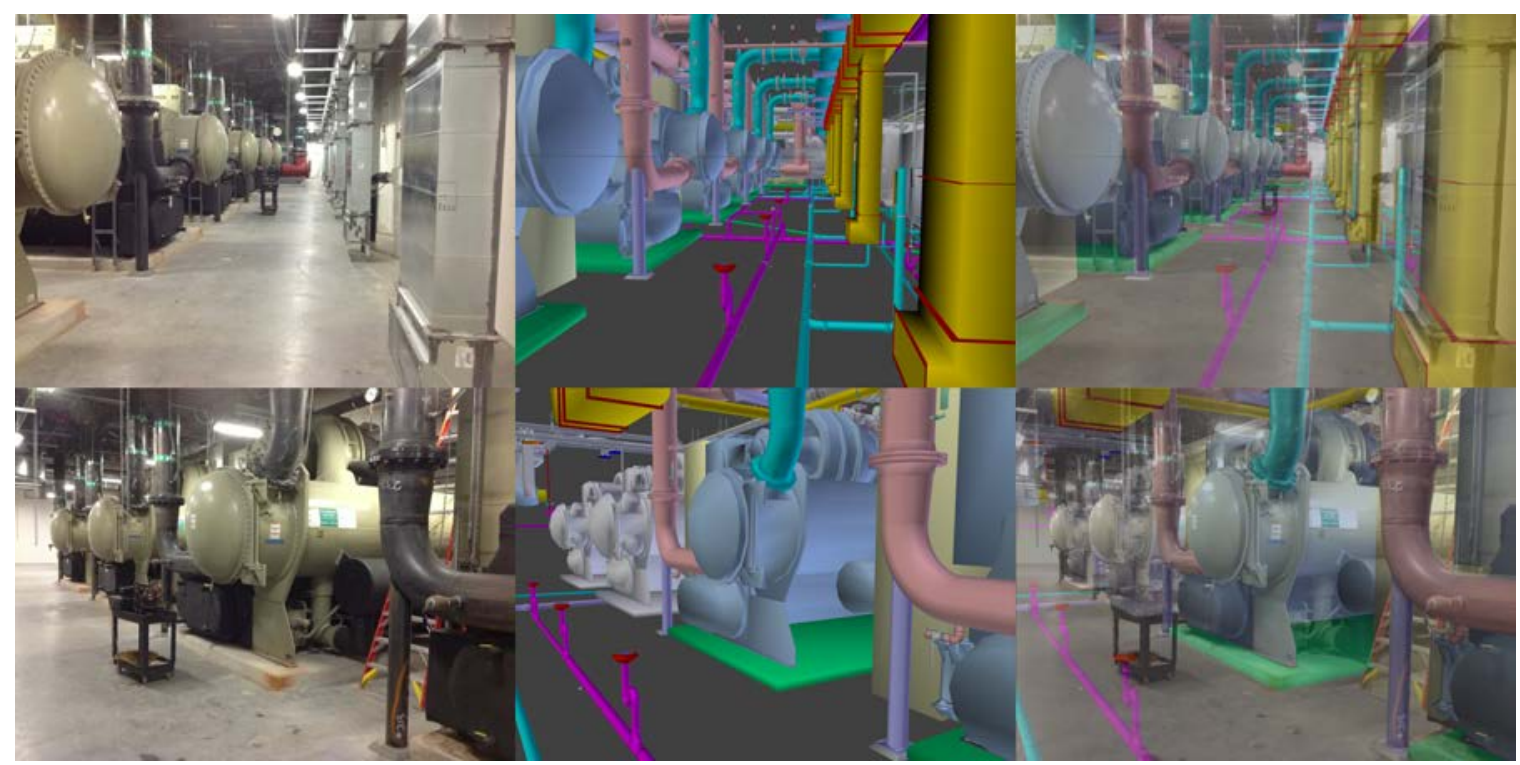

Figure 12: Images of a MEP room (left), BIM in the same view (middle), and BIM overlaid on top of the image (right). Adapted from [27]

\subsection{Capturing Progress and Condition Deviation}

Construction drawings can be very complicated, especially when comparing them to real world scenes. Figure 12 shows two typical images of a MEP room for a hospital facility taken by field personnel. Comparing the images on the left to the images on the middle, finding what parts of as-planned status do not match as-built status, and communicating any issues found are difficult. On the other hand, it is much easier to compare and spot any issues if they are shown in the same space as shown in the images on the right.

The same principle holds true for discussing progress. Showing an as-built status in 2D or 3D with a BIM is much more efficient way of communicating progress than comparing a Gantt chart with an image 

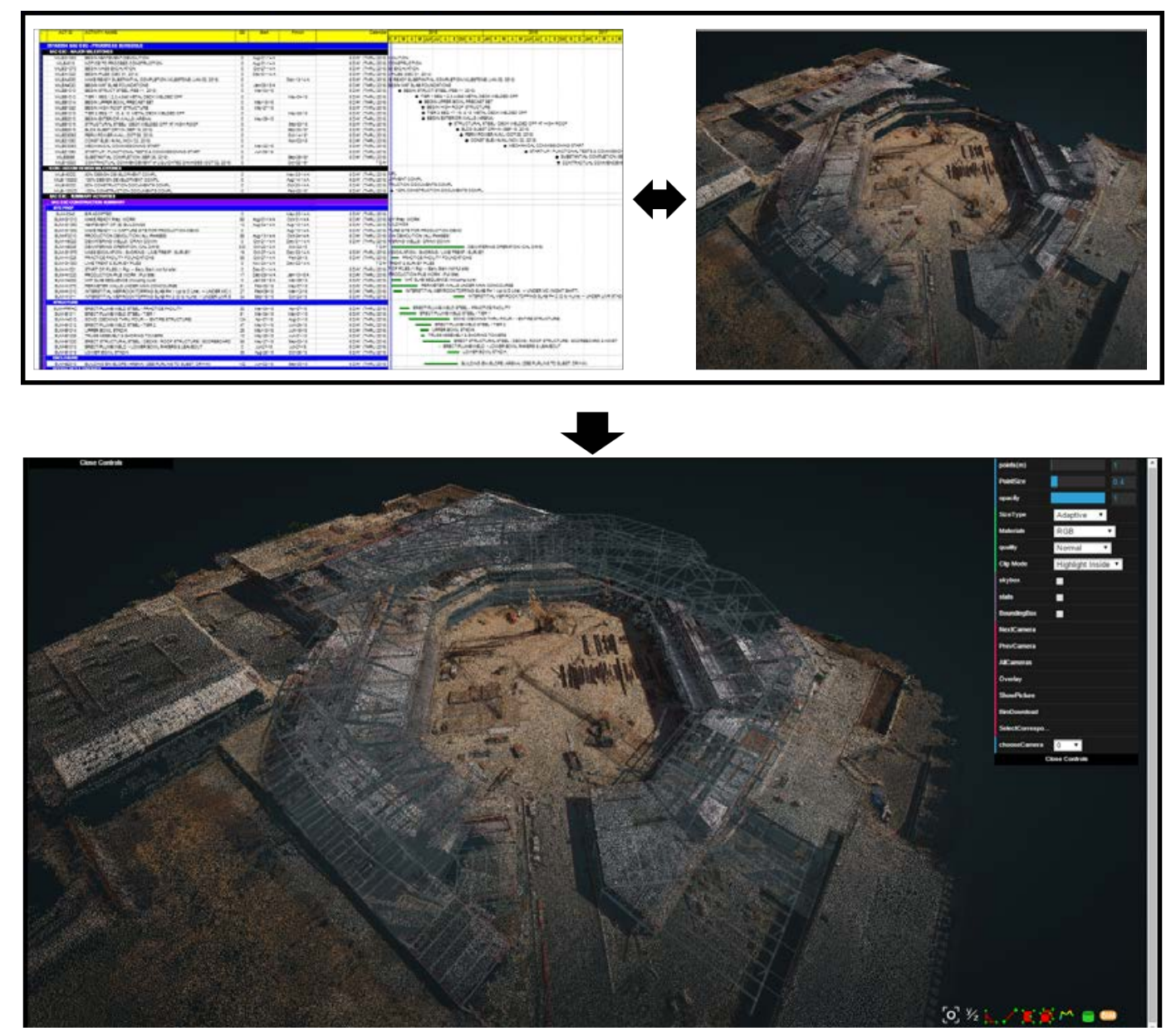

Figure 13: Comparing a schedule with as-built status (top), and progress shown in as-built and 4D BIM models superimposed together (bottom).

showing the current status, especially for non-experts and people who are not familiar with a project (see Figure 13. Figure 13 shows a point cloud and BIM superimposed through the manual selection of 3D correspondences. The images used for $3 \mathrm{D}$ reconstruction were taken by a camera-equipped UAV at a stadium project, providing aerial views that capture a wide baseline of the large structure. 926 images were captured during a flight of 20 minutes and 921 of them were registered during SfM. In other words, camera poses of 921 images are computed and they were batch-localized with the BIM. In addition to 3D-to-3D comparison as shown in the figure, producing 2D-to-3D comparisons of the 921 images similar to Figure 11 and 12 are possible.

Using BIM allows element-based selection for annotating or color-coding progress and deviations. Figure 14 consists of screenshots of a university facility. This prototype allows selection and color-coding of BIM elements. Figure 14a shows an original image of a building. Figure $14 \mathrm{~b}$ shows a cladding system selected. The selected BIM element can be colored in accordance with its progress and deviation (Figure $14 \mathrm{c} \& \mathrm{~d}$, respectively). Figure $14 \mathrm{e}$ shows the overall progress of the shown BIM elements in colors and the prototyped interface for annotation. For progress, red and green represent late and on schedule, respectively. For deviation, white indicates no deviation and blue indicates high deviation. Gradient colors between two colors (red and green for progress and blue and white for deviation) fill gradual progress and 

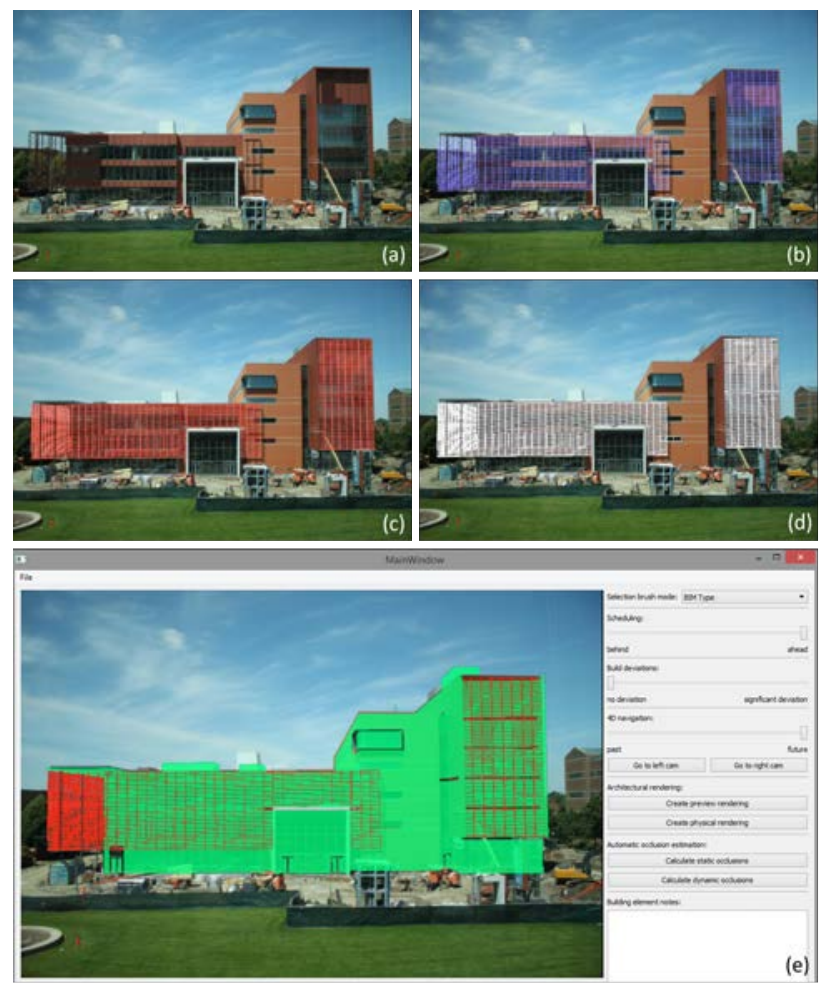

Figure 14: BIM element selection and color-coding for representing progress and deviation. (a) original image, (b) selected BIM element, color-coded for representing (c) late schedule and (d) no deviation, and (e) showing overall progress of all shown BIM elements with the developed interface.

deviation. Therefore, Figure 14e shows that the cladding system is behind schedule and all other elements are on schedule.

\subsection{Time-lapse Photorealistic 4D Rendering from Any Images}

Since multiple images taken at different times are localized, we can create time-lapse images by calculating homography matrice among images in the similar views and transforming them into a desired view. Figure 15 shows snapshots of time-lapse images from the same view, showing progress of a university facility. In this case, four images with similar views are warped and overlaid into a single view. Figure 15c can be used for annotation and documentation as discussed in the following subsection. Similarly, a 4D BIM can be rendered at any views of the localized images for creating time-lapse or simulated animations. Figure 16 shows renderings from a fixed video camera and an aerial image of a stadium project.

\subsection{Documentation}

The previous section presents how localized time-lapse photos are created. This section presents how project participants can use them in construction. Figure 17 shows an example of time-lapse photos localized with a BIM. A user can select an area of interest and navigate back and forth in time. Such feature with annotation on the image can be a useful tool for communication and documentation. A practical application would be for documenting rework and showing the states of progress before and after the rework. With this feature showing changes made at the same location, documentation for managing changes and punch lists can improve communication, especially during coordination meetings. Annotation and documentation 
features are particularly useful for MEP systems. Figure 18 illustrates an image-to-BIM localization used for documenting issues that need to be addressed. This $4 \mathrm{D}$ navigation is a feature or application that can bring performancde analyses of multiple time instances into one platform.

\section{Conclusion and Future Work}

This paper discussed the potential of big visual data used in conjunction with BIM for Construction Performance Analytics. In an effort to structure a road map for research in big visual sensing and analytics, gapsin-knowledge in current practices and research efforts are discussed. Then, insights on gaps-in-knowledge in different research areas are discussed in an effort to provide a comprehensive big picture toward which big visual data analytics is heading. After in-depth discussion on gaps-in-knowledge, a model-driven visual analytics using images and BIM as a potential solution for solving inefficient communication and poor project controls is introduced. A few potential methods for localizing visual data in construction were presented. Real world cases were used for presenting various applications that can streamline communication among all project participants, from office to field, and from field to office. Visualization of these methods together into the Integrated Project Model is an on-going research topic.

Much of the discussed methods (i.e., PnP, SfM, model-assisted SfM, scan-to-BIM registration) remain as open research challenges that need further investigation. For instance, solving a PnP problem is an approximation and there are uncertainties associated with the result. An accurate Image-to-BIM localization is not guaranteed (see Figure 19). Moreover, many other challenges discussed in Section 4 (i.e., ubiquitous interaction, object detection, and agent localization) are part of on-going research areas that need further investigation. There is not an efficient solution for dealing with a large number of visual data files. To fully implement big visual data analytics in practice, a directory structure and visualization interface for big visual data need to be investigated, which will involve filtering, sorting, and storing of visual data. 

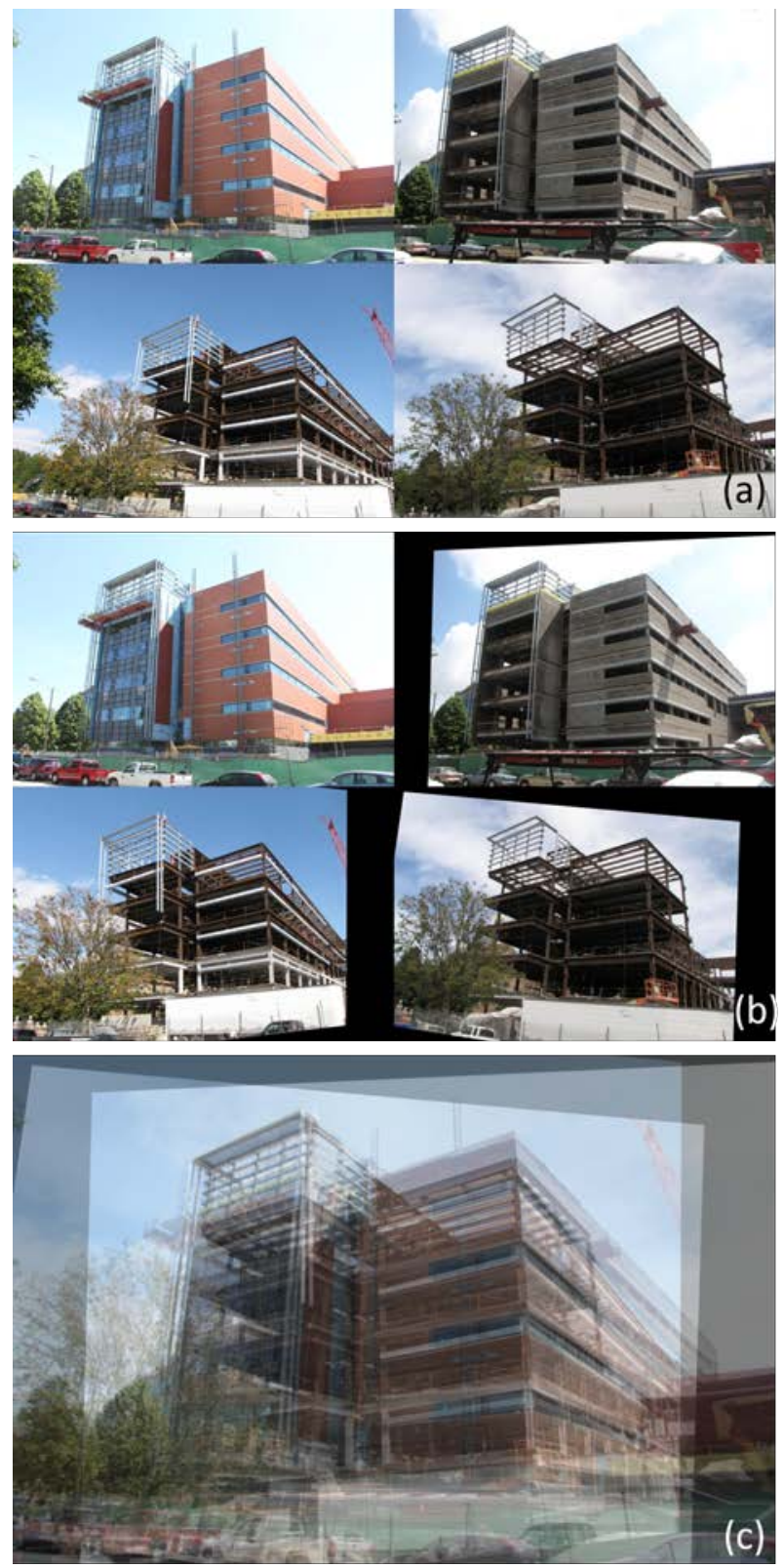

Figure 15: Creating 4D images (time-lapse): (a) original images of the similar views, (b) transformed into a single view, and (c) an overlay of all four images onto the single view. Adapted from [54] 


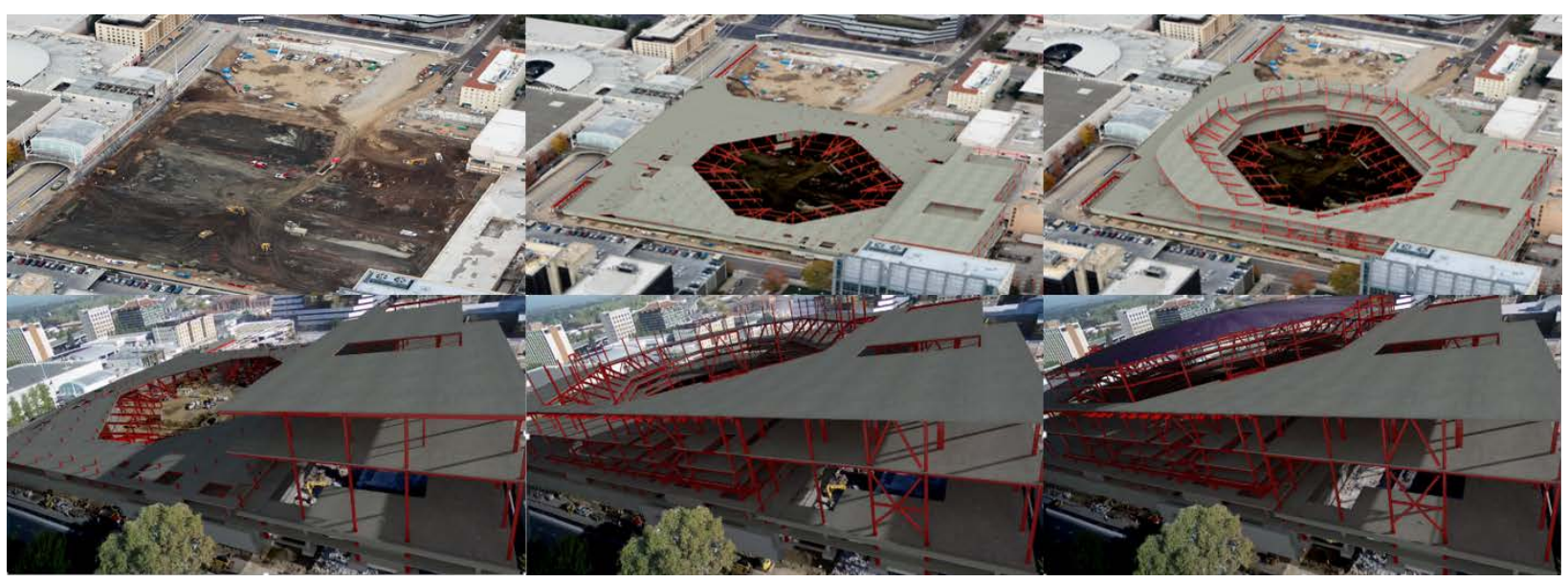

Figure 16: 4D BIM renderings: from an aerial view (top) and a fixed video camera (bottom).

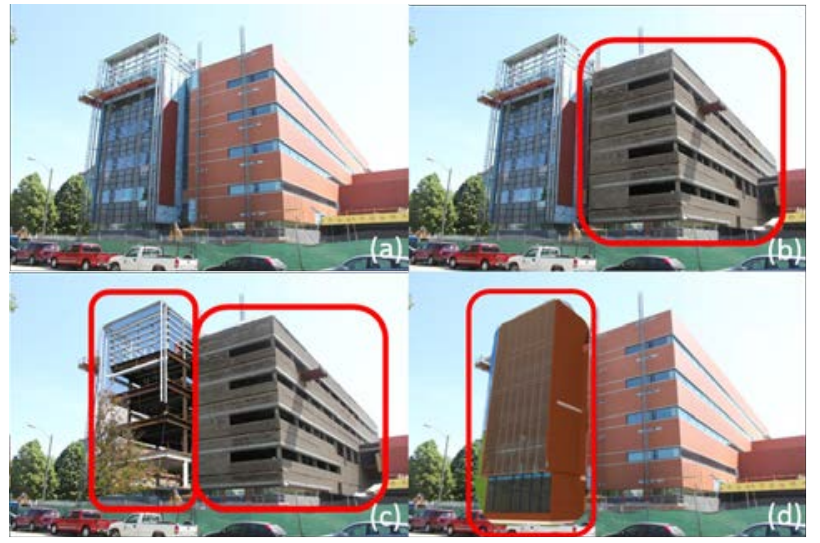

Figure 17: Time-lapse experience: (a) current time, (b) selected area 3 months ago, (c) newly selected area 4 months ago, and (d) selected area in future. Adapted from [54]

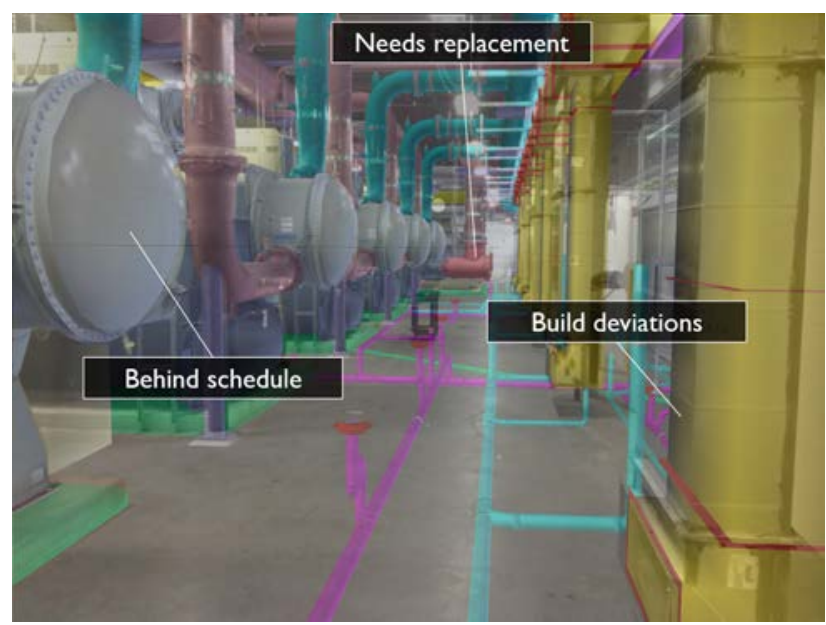

Figure 18: Annotated image-to-BIM for communicating issues. 


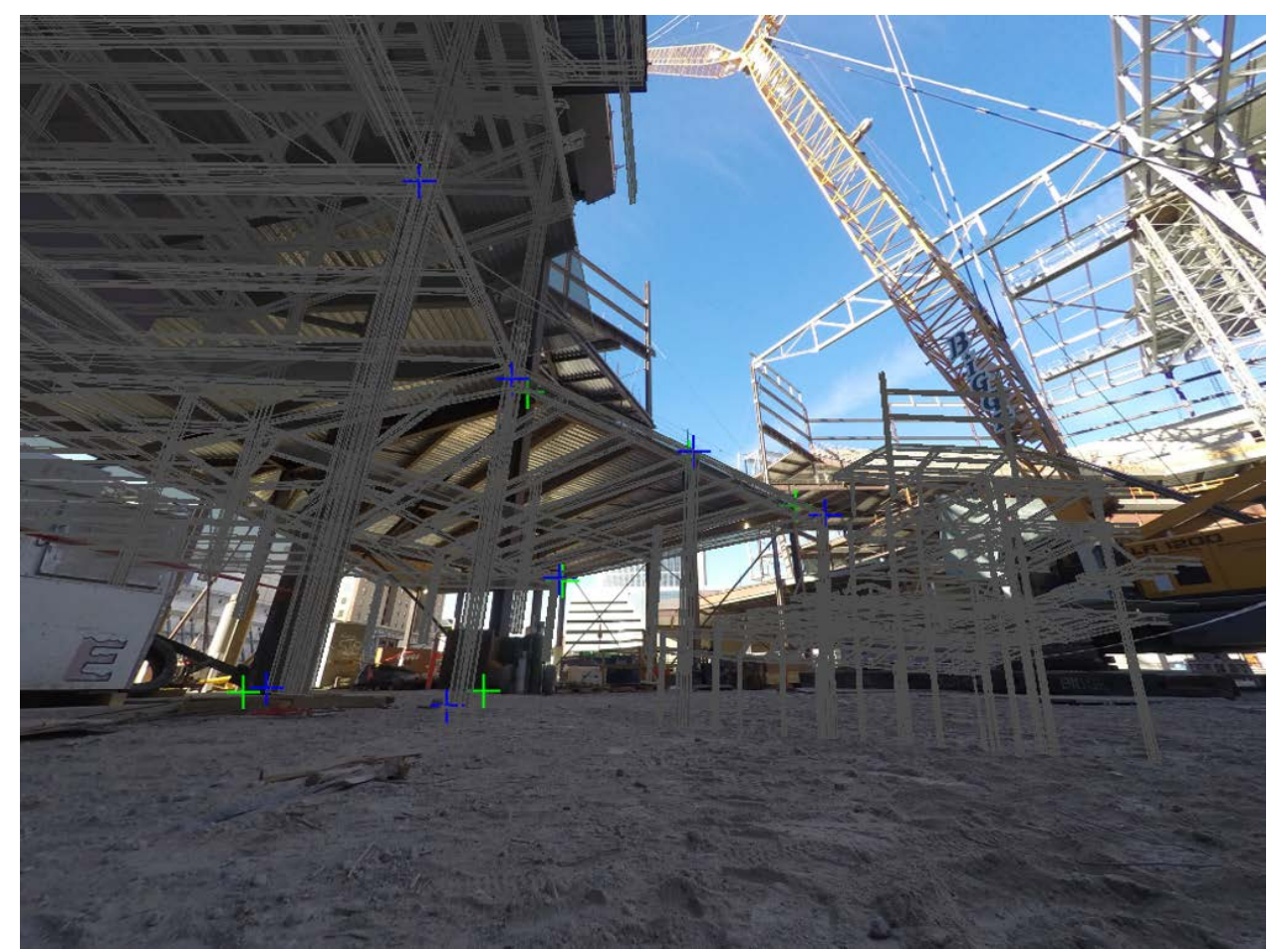

Figure 19: Misalignment after solving PnP. Green and blue crosses indicate user selections on the image and BIM, respectively. 


\section{Acknowledgment}

The authors would like to thank Turner Construction for providing access to their job sites, in addition to all the individuals who participated in the survey for this report who provided numerous constructive comments to improve it. This work is funded in part by the National Science Foundation (NSF)'s grant CMMI-1360562 and CPS-1446765 and the National Center for Supercomputing Applications (NCSA)s Institute for Advanced Computing Applications and Technologies Fellows program. Any opinions, findings, conclusions or recommendations presented in this paper are those of the authors and do not reflect the views of NSF, NCSA, Turner Construction or the individual acknowledged above.

\section{References}

[1] D. Stadnik, G. Twigg, e-mail exchange with CEO (Stadnik) and CTO (Twigg) of Multivista, Sept. 17 (2015).

[2] W. Lu, A. Fung, Y. Peng, C. Liang, S. Rowlinson, Cost-benefit analysis of building information modeling implementation in building projects through demystification of time-effort distribution curves, Building and Environment 82 (2014) 317 - 327.

[3] S. Staub-French, A. Khanzode, 3d and 4d modeling for design and construction coordination: issues and lessons learned, ITcon 12 (2007) 381-407.

[4] K. Han, M. Golparvar-Fard, The role of integrated plan and as-built models in achieving smooth flow of production in construction, in: International Conference on Innovative Production and Construction 2015, Perth, Australia, pp. 101-104.

[5] S. Ahalt, D. Bedard, T. Carsey, J. Crabtree, K. Green, C. Jeffries, D. Knowles, H. Kum, H. Lander, N. Nassar, A. Rajasekar, S. Thakur, Establishing a national consortium for data science, National Consortium for Data Science (2012).

[6] J. Liu, J. Li, W. Li, J. Wu, Rethinking big data: A review on the data quality and usage issues, \{ISPRS\} Journal of Photogrammetry and Remote Sensing (2015) -.

[7] IBM, The four vs of big data, 2013. [Online; last accessed February 14, 2015].

[8] E. McNulty, Understanding big data: The seven vs, 2014. [Online; last accessed February 14, 2015].

[9] K. Han, M. Golparvar-Fard, Appearance-based material classification for monitoring of operation-level construction progress using 4d bim and site photologs, Automation in Construction 53 (2015) 44 - 57.

[10] K. Liu, M. Golparvar-Fard, Crowdsourcing construction activity analysis from jobsite video streams, Journal of Construction Engineering and Management 141 (2015) 04015035.

[11] Web-based 4D Visual Production Models for Decentralized Work Tracking and Information Communication on Construction Sites.

[12] O. Russakovsky, J. Deng, H. Su, J. Krause, S. Satheesh, S. Ma, Z. Huang, A. Karpathy, A. Khosla, M. Bernstein, A. C. Berg, L. Fei-Fei, ImageNet Large Scale Visual Recognition Challenge, International Journal of Computer Vision (IJCV) 115 (2015) 211-252.

[13] M. Everingham, L. Van Gool, C. K. I. Williams, J. Winn, A. Zisserman, The pascal visual object classes (voc) challenge, International Journal of Computer Vision 88 (2010) 303-338.

[14] A. Dimitrov, M. Golparvar-Fard, Vision-based material recognition for automated monitoring of construction progress and generating building information modeling from unordered site image collections, Advanced Engineering Informatics 28 (2014) $37-49$.

[15] M. Golparvar-Fard, F. Peña Mora, Application of visualization techniques for construction progress monitoring, Computing in Civil Engineering (2009) 216-223.

[16] H. Bae, M. Golparvar-Fard, J. White, High-precision vision-based mobile augmented reality system for context-aware architectural, engineering, construction and facility management (aec/fm) applications, Visualization in Engineering 1 (2013) $1-13$.

[17] Y. Ham, K. K. Han, J. J. Lin, M. Golparvar-Fard, Visual monitoring of civil infrastructure systems via camera-equipped unmanned aerial vehicles (uavs): a review of related works, Visualization in Engineering 4 (2016) 1-8.

[18] J. Abeid, D. Arditi, Time-lapse digital photography applied to project management, Journal of Construction Engineering and Management 128 (2002) 530-535.

[19] J. Abeid, E. Allouche, D. Arditi, M. Hayman, Photo-net ii: a computer-based monitoring system applied to project management, Automation in Construction 12 (2003) 603 - 616. Computer Aided Architectural Design Research in Asia.

[20] J. S. Bohn, J. Teizer, Benefits and barriers of construction project monitoring using high-resolution automated cameras, Journal of Construction Engineering and Management 136 (2009) 632-640.

[21] M. Golparvar-Fard, F. Peña Mora, C. A. Arboleda, S. Lee, Visualization of construction progress monitoring with 4d simulation model overlaid on time-lapsed photographs, Journal of Computing in Civil Engineering 23 (2009) 391-404. 
[22] C. Woodward, K. Kähkönen, J. Hyväkkä, P. Janne, S. Siltanen, Integrating building product models with live video stream, in: Proc., 7th Int. Conf. on Construction Applications of Virtual Reality, pp. 176-188.

[23] D. Rebolj, N. Čuš Babič, A. Magdič, P. Podbreznik, M. Pšunder, Automated construction activity monitoring system, Advanced Engineering Informatics 22 (2008) 493 - 503.

[24] R. I. Hartley, A. Zisserman, Multiple View Geometry in Computer Vision, Cambridge University Press, 2 nd edition, 2004.

[25] D. Lowe, Distinctive image features from scale-invariant keypoints, International Journal of Computer Vision 60 (2004) 91-110.

[26] J. Yang, M.-W. Park, P. A. Vela, M. Golparvar-Fard, Construction performance monitoring via still images, time-lapse photos, and video streams: Now, tomorrow, and the future, Advanced Engineering Informatics (2015).

[27] K. Karsch, M. Golparvar-Fard, D. Forsyth, Constructaide: Analyzing and visualizing construction sites through photographs and building models, ACM Trans. Graph. 33 (2014).

[28] J. DeGol, D. Hanley, N. Aghasadeghi, T. Bretl, A passive mechanism for relocating payloads with a quadrotor, International Conference on Intelligent Robots and Systems (2015).

[29] B. K. P. Horn, Closed-form solution of absolute orientation using unit quaternions, Journal of the Optical Society of America A 4 (1987) 629-642.

[30] M. Golparvar-Fard, F. Peña Mora, S. Savarese, Application of d4ar a 4-dimensional augmented reality model for automating construction progress monitoring data collection, processing and communication, ITcon 14 (2009) 129-153.

[31] M. Golparvar-Fard, F. Peña Mora, S. Savarese, Automated progress monitoring using unordered daily construction photographs and ifc-based building information models, Journal of Computing in Civil Engineering (2012) 147.

[32] P. Tang, D. Huber, B. Akinci, R. Lipman, A. Lytle, Automatic reconstruction of as-built building information models from laser-scanned point clouds: A review of related techniques, Automation in Construction 19 (2010) 829-843.

[33] F. Bosché, Plane-based registration of construction laser scans with 3d/4d building models, Advanced Engineering Informatics 26 (2012) 90-102.

[34] M. Nahangi, C. Haas, J. West, S. Walbridge, Automatic realignment of defective assemblies using an inverse kinematics analogy, Journal of Computing in Civil Engineering 0 (0) 04015008.

[35] J. Lin, K. Han, Y. Fukuchi, M. Eda, M. Golparvar-Fard, Model-based monitoring of work-in-progress via images taken by camera-equipped uav and bim, in: Proc. of the 2nd International Conference on Civil and Building Engineering Informatics, Tokyo, Japan.

[36] F. Bosché, Automated recognition of $3 \mathrm{~d}$ cad model objects in laser scans and calculation of as-built dimensions for dimensional compliance control in construction, Advanced Engineering Informatics 24 (2010) 107-118.

[37] F. Bosché, A. Guillemet, Y. Turkan, C. Haas, R. Haas, Tracking the built status of mep works: Assessing the value of a scan-vs-bim system, Journal of Computing in Civil Engineering (2013).

[38] F. Bosché, C. T. Haas, B. Akinci, Automated recognition of 3d cad objects in site laser scans for project 3d status visualization and performance control, Journal of Computing in Civil Engineering 23 (2009) 311-318.

[39] C. Kim, B. Kim, H. Kim, 4d $\{\mathrm{CAD}\}$ model updating using image processing-based construction progress monitoring, Automation in Construction 35 (2013) $44-52$.

[40] K. Han, D. Cline, M. Golparvar-Fard, Formalized knowledge of construction sequencing for visual monitoring of work-inprogress via incomplete point clouds and low-lod 4d bims, Advanced Engineering Informatics (2015).

[41] S. Daum, A. Borrmann, Processing of topological $\{$ BIM $\}$ queries using boundary representation based methods, Advanced Engineering Informatics 28 (2014) 272 - 286.

[42] Characterization of laser scanners and algorithms for detecting flatness defects on concrete surfaces, Journal of Computing in Civil Engineering 25 (2011) 31-42.

[43] M.-K. Kim, J. C. Cheng, H. Sohn, C.-C. Chang, A framework for dimensional and surface quality assessment of precast concrete elements using \{BIM\} and 3d laser scanning, Automation in Construction 49, Part B (2015) 225 - 238. 30th \{ISARC\} Special Issue.

[44] K. Han, J. Gwak, M. Golparvar-Fard, K. Saidi, G. Cheok, M. Franaszek, R. Lipman, Vision-based field inspection of concrete reinforcing bars, 13th International Conference on Construction Applications of Virtual Reality (2013) 272-281.

[45] S. Du, M. Shehata, W. Badawy, Hard hat detection in video sequences based on face features, motion and color information, in: Computer Research and Development (ICCRD), 2011 3rd International Conference on, volume 4, pp. 25-29.

[46] S. Han, S. Lee, F. Peña Mora, Vision-based detection of unsafe actions of a construction worker: Case study of ladder climbing, Journal of Computing in Civil Engineering 27 (2013) 635-644.

[47] S. Han, S. Lee, A vision-based motion capture and recognition framework for behavior-based safety management, Automation in Construction 35 (2013) $131-141$.

[48] S. Chi, C. H. Caldas, Automated object identification using optical video cameras on construction sites, Computer-Aided Civil and Infrastructure Engineering 26 (2011) 368-380.

[49] M. Golparvar-Fard, A. Heydarian, J. C. Niebles, Vision-based action recognition of earthmoving equipment using spatio- 

temporal features and support vector machine classifiers, Advanced Engineering Informatics 27 (2013) 652 - 663.

50] M. Memarzadeh, M. Golparvar-Fard, J. C. Niebles, Automated 2d detection of construction equipment and workers from site video streams using histograms of oriented gradients and colors, Automation in Construction 32 (2013) 24 - 37.

[51] J. Wang, S. Zhang, J. Teizer, Geotechnical and safety protective equipment planning using range point cloud data and rule checking in building information modeling, Automation in Construction 49, Part B (2015) 250 - 261. 30th \{ISARC\} Special Issue.

[52] E. Marks, T. Cheng, J. Teizer, Laser scanning for safe equipment design that increases operator visibility by measuring blind spots, Journal of Construction Engineering and Management 139 (2013) 1006-1014.

[53] S. Chi, C. Caldas, Image-based safety assessment: Automated spatial safety risk identification of earthmoving and surface mining activities, Journal of Construction Engineering and Management 138 (2012) 341-351.

[54] K. K. Han, M. Golparvar-Fard, BIM-Assisted Structure-from-Motion for Analyzing and Visualizing Construction Progress Deviations through Daily Site Images and BIM, pp. 596-603. 